\title{
Novel Method of Capturing Static and Dynamic Anthropometric Data for Home Design
}

\author{
Pin Zhang ${ }^{+}$, Shengfeng Qin ${ }^{*}$, and David $\mathrm{K}$ Wright, ${ }^{*}$ Member, IEEE
}

\begin{abstract}
This paper presents a nowel method for capturing and measuring both static and dynamic anthropometric data of people. These data can be then used for bartier-free home design based on a concept of ergonomic design with motion. This new approach utilized a 3D motion capture system as a tool to simultaneously obtain anthropometric information based on body motion analyses. This paper reports the experimental system design, data collection and analysis techniques on body motions.
\end{abstract}

Keywords - anthropometric data, dynamic information accuisition, ergonomic design, motion analysis.

\section{INTRODUCTION}

$\mathrm{T}$ HE number of elderly people is markedly increasing in developed and developing countries such as the UK and China. In general, elderly people will have some biologic and physical degeneration when they become aged. These may result in the loss of physiological function, exercise ability or mobility (such as the body atrophy, reduction in hearing and visual impaiment, slowmoving, and reduced range of motion). Moreover, the degeneration may be also caused in various degrees by some diseases, e.g, heart disease, high blood pressure, arthritis and diabetes. With decreasing mobility, elderly people find more and more difficulties in their daily life. Therefore, in order to improve their quality of life, it is very important to investigate home design using ergonomics principles for the handicapped and people with special needs and for the frail elderly $[1,2]$. For example, in order to make it easy to use bathroom facilities safely and conveniently, we should design these facilities such as bathtub for them in accordance with the their anthropometry and mobility.

To do so, knowledge about human body size and anthropometric data of elderly people is essential, but not alone sufficient for the ergonomic design because the

S. F. Qin. is with the School of Enginering \& Design, Brunel University, Middlesex, Uxbridge, UK; (phone: +44-1895-266335 fax: +44-1895-269763; e-mail: sheng.feng.Qin@brunel.ac.uk)

P. Thang is with the College of Arts and Design, Tianjin University of Science and Technology, Tianjin 300222, China (email:pzh1959@yahoo.com).

D. K. Wright, is with the School of School of Engineering \& Design, Brunel Univer sity, Middlesex, Uxbridge, UK; (phone: +44-1895-65770; e-mail: david.wright@brunel.ac.uk). standard anthropometric measures are static. In the real world, human interactions with the physical world include many dynamic features and information. Furthermore, there is, in general, a lack of adequate anthropometric data on the elderly. For example, we only know the standard basic size of human body for Chinese adults (male 18-60, female 18-55) from Chinese National Standard Documents (GB1000-88), while the specific data for Chinese aged people is not available. Although some research $[3,4]$ has shown that the body size of old people can be derived from those of mature adult sizes, we still prefer to have experimental tests to obtain a set of new data reflecting on both body size and dynamic mobility, because the published body size is static data, not reflecting changes in mobility $[5,6]$.

Traditional method to obtain anthropometric data is to survey characterized populations (the use of averages and percentiles). This method has difficulty in measuring anthropometric data against multiple realistic-postures. The 3D scanning techniques (http//store.sae.org/caesar/) have been recently used to capture 3-D data of subjects in realistic postures allowing to extract an almost infinite number and variety of measurements long after the subject has moved. However, these methods can only capture static data.

Thus, it is novel to develop a method for simultaneously measuring both static and dynamic anthropometric data for product and home design applications. This paper describes the method and its experimental system design in Section 2. Section 3 introduces data collection and analysis techniques based on body motions. Some test results for bathing facilities design are discussed in Section 4 , and finally, conclusions are drawn.

\section{THEMETHOD AND ITS EXPERIMENTAL SYSTEM}

The human body size and mobility measurement method using a 3D motion capture system includes three stages: measurement scene setting up, marker design and motion capturing, and motion data processing and interpretation. This section introduces first two stages.

\section{A. Measurement Scene Setting Up}

The concept of ergonomic design with motion was realized using a motion capturing system by Motion Analysis [7]. It is a 3D optical motion capturing system that measures the movement of objects. The system combines proprietary hardware, software and electrooptical techniques with standard computer and video 
hardware. In our system, 7 Eagle digital cameras were used with the EVaRT 4.2 software to collect motion data. The 7 cameras can be located circularly to let the system to capture body motions within a rough volume about $5 \mathrm{~m} \mathrm{X}$ $8 \mathrm{~m} \mathrm{X} 4 \mathrm{~m}$ along the $x, y$ directions on the ground and the vertical $z$ direction. Motion is captured from reflective markers. The size of marks varies from $6 \mathrm{~mm}$ to $25 \mathrm{~mm}$ in diameter. Different sizes of markers will affect the size of the volume. After setting up the motion capturing system, a test scene with various $3 \mathrm{D}$ references was set up in the centre of the capturing region. For example, an elastic string had been suspended horizontally with the foot of tables (Fig. 1). Its height could be changed easily to test people's motion of crossing the string line (similar to the edge of bathtub). The use of the string can reduce the obstruction of view from cameras. On the ground, we used white paper tapes to mark a base of the 'bathtub'. One foot of the table on the right side is close to the centre, which allows people to use it as an assistance handle if needed. These references were visible, touchable and pressuresensible.

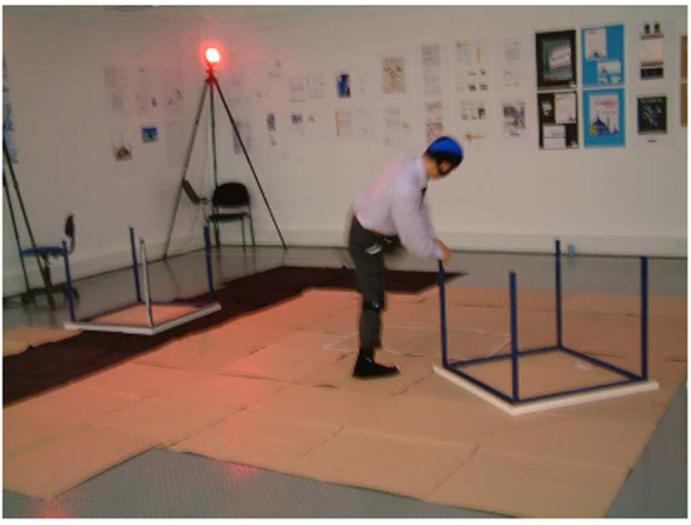

Figure 1. Test scene design

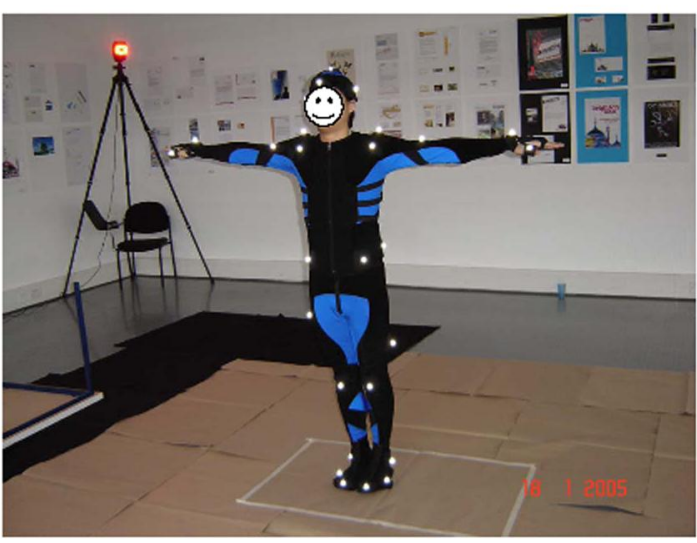

Figure 2. Marker design

\section{B. Marker Design and Motion Capture}

In order to measure the body size and mobility correctly, a good marker design is required. How many markers we need depends on applications. For a general purpose, the marker design can refer to Helen Hayes Marker Set
Placement

(http://www.lifemodeler.com

LM_Manual/A_motion.htm) (Fig.2). When a subject wearing designed markers moves in the test scene, the corresponding motion can be captured.

\section{MOTION DATA PROCESSING AND INTERPRETATION}

After receiving motion data captured by the system, the motion was recorded in a Track Row Column (.TRC) file, which contains $X-Y-Z$ position data for the reflective markers. The file is in the ASCII format that can be easily read into a spreadsheet program such as Excel ${ }^{\mathrm{TM}}$. The position data for each marker is organized into 3 columns per parker ( $\mathrm{X}, \mathrm{Y}$ and $\mathrm{Z}$ position) with each row being a new frame. The position data is relative to the global coordinate systems of the capture volume and the position values are in the units used for calibration. Missing data is represented as an empty frame of position data.

In a TRC file, the first row will represent a starting pose. Analyzing data extracted from this row against mark settings can result in static anthropometric data such as heights, shoulder widths, lower leg lengths. Table 1 shows an example of 11 people's static data extracted from a motion capture file. Each row represents one person's static dimensions such as heights of forehead, shoulder, elbow, knee, heel and toe.

TABLE 1: STATIC DATA SHEET.

\begin{tabular}{|c|c|c|c|c|c|}
\hline F, Head & Shoulder & elboww & knee & Heel & Toe \\
\hline 1617.513 & 1361.833 & 1045.782 & 497.9818 & 59.92479 & $\begin{array}{l}42.5558 \\
8\end{array}$ \\
\hline 1537.54 & 1242.22 & 984.1884 & 525.8735 & 54.88779 & $\begin{array}{l}44.8343 \\
2\end{array}$ \\
\hline 1676.67 & 1397.395 & 1122.163 & 466.7862 & 95.55046 & $\begin{array}{l}52.8123 \\
1\end{array}$ \\
\hline 1647.7 & 1442.348 & 1141.566 & 468.2026 & 59.73159 & $\begin{array}{l}49.4354 \\
2\end{array}$ \\
\hline 1716.527 & 1420.328 & 1173.16 & 504.0228 & 62.19487 & $\begin{array}{l}49.6867 \\
8\end{array}$ \\
\hline 1471.483 & 1240.019 & 960.6669 & 440.757 & 48.93247 & 47.6213 \\
\hline 1699.29 & 1433.228 & 1153.37 & 462.5336 & 62.38312 & $\begin{array}{l}47,4173 \\
1\end{array}$ \\
\hline 1433.701 & 1261.405 & 1092.011 & 535.8144 & 75.24448 & $\begin{array}{l}18.8200 \\
6\end{array}$ \\
\hline 1540.088 & 1290.282 & 985.0888 & 447.8157 & 49.77518 & $\begin{array}{l}40.3664 \\
4\end{array}$ \\
\hline 1580.646 & 1402.299 & 1066.349 & 458.0783 & 61.4655 & $\begin{array}{l}59.6095 \\
2\end{array}$ \\
\hline 1510.404 & 1288.203 & 1030.523 & 405.3123 & 129.0469 & $\begin{array}{l}122.592 \\
9\end{array}$ \\
\hline
\end{tabular}

From motion data, the EVaRT 4.2 software can be used to analyze dynamic information based on the relation of markers and segments (See Fig. 3). Velocity of each marker can be obtained by computing positional displacement per frame. Acceleration of a marker is based on velocity difference per frame. The size of a segment can be measured as a distance between two marks. Included angles between two body segments can be calculated by selecting two lines with 4 (or 3 ) markers. The velocity and acceleration information can be further interpreted to reflect easiness and confidence of performing a specific task. The included angles also indicate the range of motion required for a task. Their variations reflect changes of mobility. All these dynamic information is very useful 
for ergonomic studies in product and home design applications.

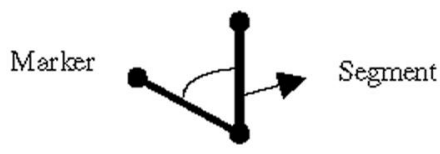

Figure 3. Relation of markers and segments

\section{TESTING RESULTS}

In order to test our new method, we randomly selected 20 volunteers with different genders, ages and places of birth. The data collection process was associated to a home design (a bathtub) for the elderly. The goal was to identify dynamic features when people crossed the edge of a 'bathtub' (string).

The test scene was shown in Figure 1. The test heights were set to $400 \mathrm{~mm}, 450 \mathrm{~mm}, 500 \mathrm{~mm}, 550 \mathrm{~mm}$, and $700 \mathrm{~mm}$. In the test, the volunteers were required to be barefoot. At the beginning of each test, they gave a standard pose (Fig. 2) to show their body size rightly. Then, they were asked to feel free and natural to 'walk' into the bathtub (walking crossing the string) and get out the bathtub (walk back). The whole motion was captured.

Some static data collected from this test has been given in the Table 1 . The body sizes were measured correctly.

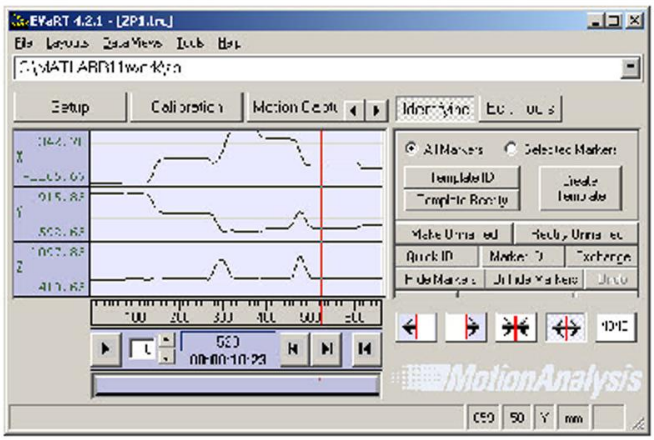

Figure 4a. Dynamic information: position graph

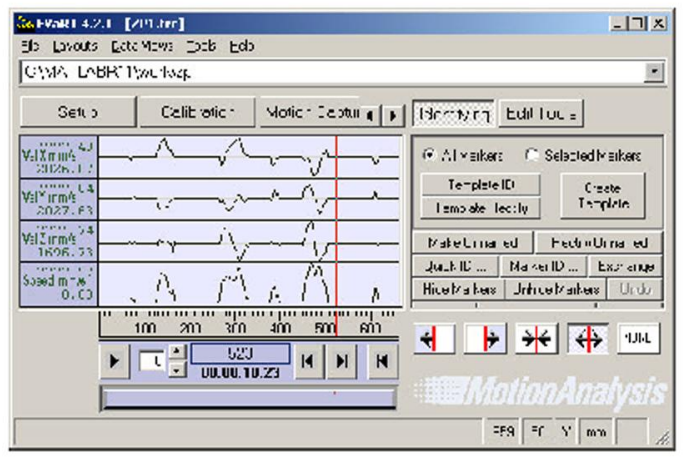

Figure 4b. Dynamic information: velocity graph

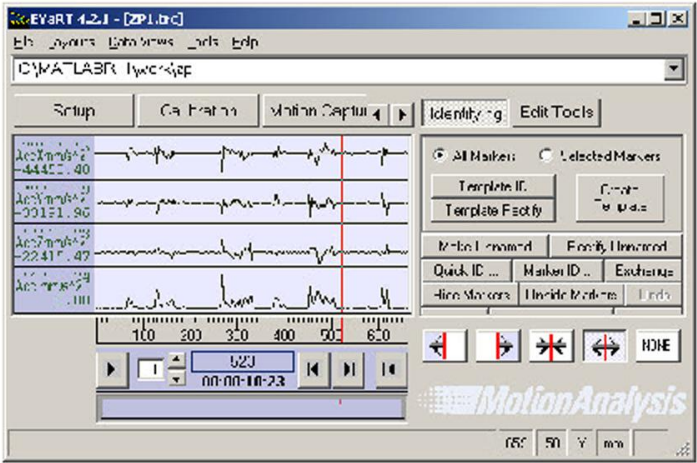

Figure 4c. Dynamic information: acceleration

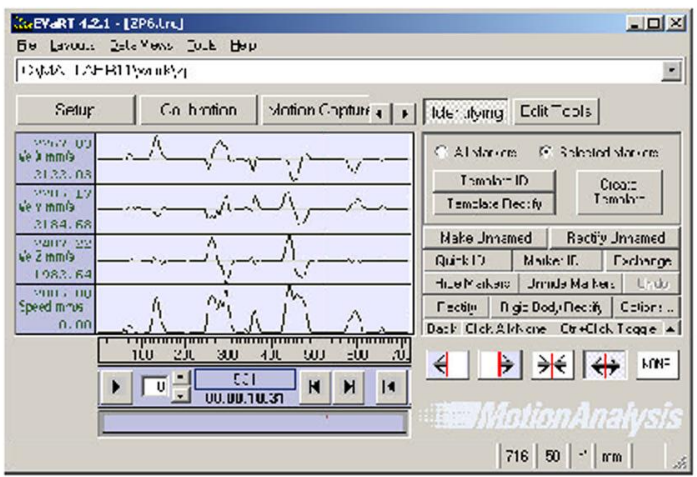

Figure 4d. Dynamic information: velocity graph for another

Figure 4 gives dynamic information with graphs. The figures $4 a$ and $4 b$ indicate the position and velocity changes of a selected marker when a person crossed the string (height $=500 \mathrm{~mm}$ ) and came back. The corresponding acceleration graph is shown in Figure $4 c$. The graph in Figure $4 \mathrm{~d}$ describes the velocity changes of another person crossing the string (height $=700 \mathrm{~mm}$ ). Comparing different people's motion graphs, it is clear that there are some certain patterns corresponding to the crossing motion. By studying the acceleration curves, a force and a moment for crossing the bathtub can be computed later.

In order to compare the performances between two subjects and identify degenerations of aged people, we selected two men with a similar height but different ages to cross the bathtub at the height of $700 \mathrm{~mm}$. This setting was lower than the average bathtub height of $750-800 \mathrm{~mm}$ in China. Figure 4 shows performance curves from a 23 years old subject (4a: position; 4b: velocity; 4c: acceleration). The counterpart's performance from a 50 years old subject is given in Fig 5 correspondingly. From them, it was observed that the ranges of speed $(0-3834.4)$ and acceleration (0-32120.44) from the young were nearly half of those (speed: 0-5310.60; acceleration: 0-57245.01) from the old. This information can be interpreted that old people have to gain a big moment with high-speed motion to cross the bathtub. This indicates that aged people have less confidence in their mobility even when you can physically perform a movement. This degeneration was further evidenced by the time interval between starting the 
crossing (corresponding to a small wave just before a big wave) and reaching the peak of the crossing. The young man used 87 seconds while the old 105 . That is to say, the old people may had psychological barrier to cross the string and needed more time to thinking. Of course, the dynamic information needs to be studied fully to support the design. It is obvious that the $700 \mathrm{~mm}$ height of a bathtub needs to be reduced.

\section{CONCLUSION}

A 3D motion capturing system as a tool can be used to simultaneously obtain both static and dynamic anthropometric data of elderly people for ergonomics study and design. The method can capture task-specific dynamic information related to mobility and physiological degenerations. Motion data allows us to extract a great number and variety of measurements long after the subject has moved. It is very useful for ergonomic design applications. The experimental results from the case studies will be reported separately.

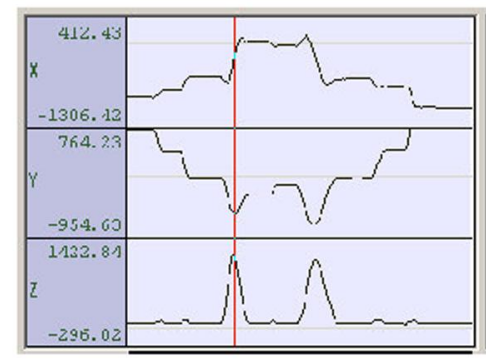

Figure 5a. Performance of a young subject (23yrs old): position graph

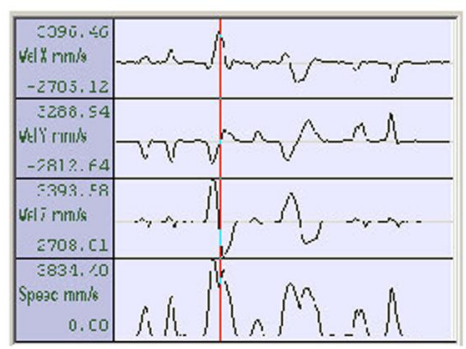

Figure 5b. Performance of a young subject (23yrs old): position graph

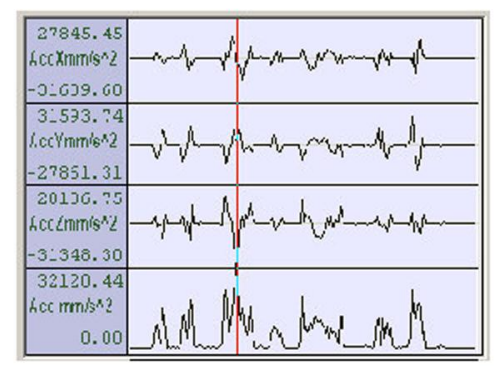

Figure 5c. Performance of a young subject (23yrs old): position graph

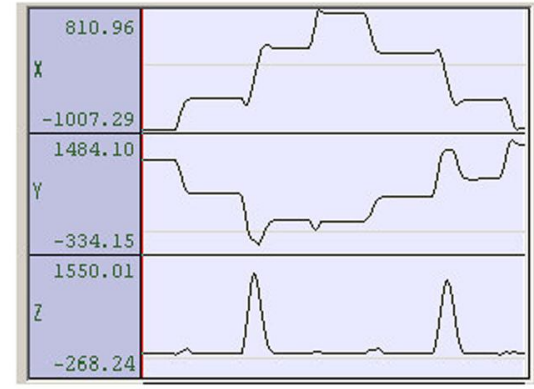

Figure 6a. Performance of a middle-aged subject (50yrs old): position graph

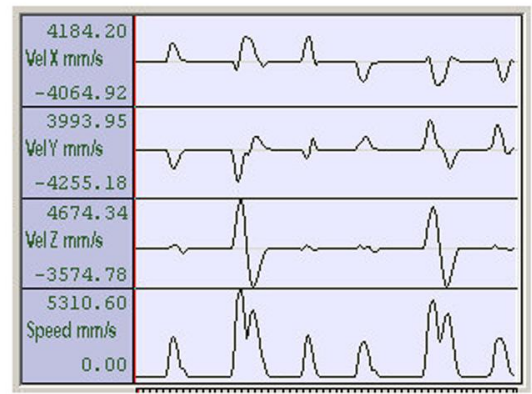

Figure 6 . Performance of a middle-aged subject (50yrs old): velocity graph

\begin{tabular}{|c|c|c|}
\hline Acco202.97 \\
-42073.46
\end{tabular}

Figure 6c. Performance of a middle-aged subject (50yrs old): acceleration graph.

\section{REFERENCES}

[1] M. Farquhar, Edlerly peoples's definitins of quality of life, Soc. Sci. Med. Vol, 41(10), pp.1439-1446, 1995:

[2] O. Demirbilek, H. Demirkan, Universal product desing involving elderly users: a participatory design model, Applied Ergonomics Vol. 35, pp.361-370.2004

[3] H. Klingbeil, et al. Aging with a disability, Arch Phys Med Rehabil Vol. 85(suppl 3), pp. \$68-73,2004.

[4] M.R. Pinto, et al, Eromomics, Gerontechnology, and design for the home-environment : Applied Ergonomics Vol.31, pp. 317-322, 2000 .

[5] K. Kothiyal, T. Tttey, Anthropomefric data of elderly people in Australia, Applied Ergonomics Vol.31, pp.329-332, 2000.

[6] K. Heli, V. Seppo, H. Ari, Three evaluations of task-surface heights in elderly people's homes, Applied Ergonomics Vol. 31, PP. 109. 119,2000 .

[7] http:/www.motionanalysis.com 